\title{
Research on User Online Learning Behavior Analysis Model in Big Data Environment
}

\author{
Zhang Guoming \\ Nanchang Institute of Science \& Technology, Nanchang, 330108, China
}

Keywords: learning behavior; analysis model; data mining; online learning; big data

\begin{abstract}
With the advent of the era of large data, the continuous emergence of mass learning data needs to be processed with new methods and tools, and the analysis of online user learning behavior has emerged as the times and has become a hot issue in the field of educational research and application. In order to improve the efficiency of online learning behavior analysis in large data environment, the current analysis techniques and tools of learning behavior are analyzed. The analysis shows that the main analytical techniques include data mining, statistics and mathematical methods, and the main analysis model is process analysis model and continuous upgrading cycle model. Three models, such as model and behavior analysis cycle model, are put forward in the end of the learning behavior analysis model and method technology, which are of great significance for the intervention of online learning behavior and the optimal allocation of learning resources.
\end{abstract}

\section{Introduction}

Since twenty-first Century, with the development of mobile Internet technology, cloud computing, and Internet of things technology, big data came into being, and its research and application have been developing continuously. Before the advent of online learning and cloud computing, people have begun to collect, analyze and utilize educational data. In 1990s, in the study of the intelligent tutor system (ITS), researchers and designers have begun to apply complex methods of learning analysis, which are used in a later online learning environment. With the increasing number of online learning users, the intervention of online user learning methods and the optimal allocation of learning resources have become a hot issue in the current study of learning behavior analysis. How to improve the efficiency of online user learning behavior analysis is of great significance for improving the user's individualized self-adaptive learning level.

\section{The learning behavior analysis techniques and use tools}

At present, there are many methods for learning behavior analysis technology, among which two kinds of learning behavior data mining and statistical and mathematical methods are the mainstream. There are also many tools for learning behavior analysis. This time introduces two common methods, including personalized analysis tools and learning analysis tools supporting educational decision-making.

\subsection{The data mining of learning behavior.}

One of the most important methods for the analysis of learning behavior is the mining of learning behavior data. The online learning behavior of users will produce massive learning data. The method of data mining can be used to analyze the learning behavior and learning results of users, such as multiple linear regression algorithm, clustering analysis algorithm and decision tree. These methods can be used to evaluate the performance of online learning, the relationship between learning influence factors and the problems of user learning behavior, and provide an effective reference for optimizing online learning resources.

\subsection{The statistical and mathematical methods.}

Statistical and mathematical methods are the basic methods for the analysis of learning behavior 
data, such as median, standard deviation and mean value. General statistics are used together with mathematical methods and data mining methods. It is possible to establish a user's online learning behavior feature summary, scoring system, and intelligent learning system.

\subsection{The text analysis and semantic analysis.}

Text analysis can transform text data into information that computer can recognize and process, thus improving the efficiency of text processing. Semantic analysis uses semantic principles to improve the quality of data processing and to identify the meaning of data more accurately.

\subsection{The personalized online learning and behavioral analysis tools.}

Personalized online learning and analysis tools, on the one hand, can provide learning resources for online learning users. On the other hand, learning behavior data can be tracked and behavioral data are presented to students in the visual form, thus helping online learning users to choose learning resources according to their own learning conditions.

\subsection{The behavioral analysis tool to support educational decision making.}

Through the collection and processing of a large number of educational data, the learning analysis tool that supports educational decision-making provides a scientific and rigorous research approach and more convincing decision basis for the education researchers or decision-makers through the collection and processing of a large number of educational data.

\section{The analysis model of online learning behavior based on large data}

In the background of large data, users can contact a large number of learning resources through the network platform. Online learning has a growing impact on the behavior of the learners, and the analysis of learning behavior has also begun to focus on the user's online learning behavior. The analysis of user online learning behavior can use intelligent data, user learning behavior and analysis model to discover the relationship between the learner's behavior and information and the society, and make a prejudgement and suggestion on the online learning behavior of the user. The process model is shown in Figure 1.

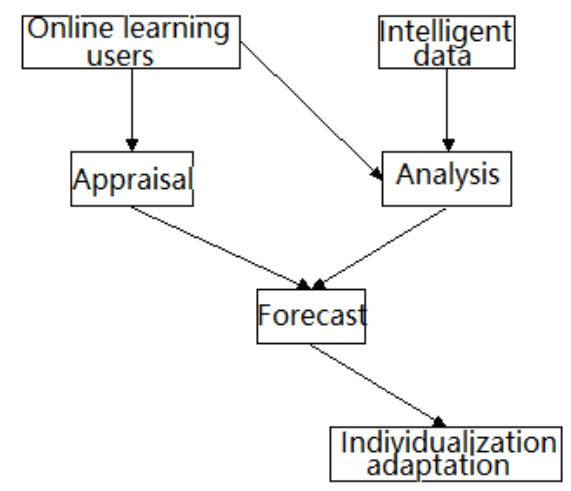

Fig.1 The user online learning behavior analysis process model

As shown in Figure 1, the main purpose of the online learning behavior analysis is to predict, manage and intervene in the learning process, to provide the learners with personalized learning resources, to improve the self-adaptive ability of the learning process, to realize the individualization and adaptation of the learning process, and to combine the network technology, education and society. Prime.

The continuous promotion model of learning behavior analysis is an analysis model combining four aspects of resources, including computer, manpower, organization and theory, which can be used for data collection, information processing, and knowledge utilization for online learning behavior. Finally, the analysis results of users' online behavior can be shared so that the university can expand the University. The role of learner behavior analysis promotes a new round of learning analysis and optimization. 


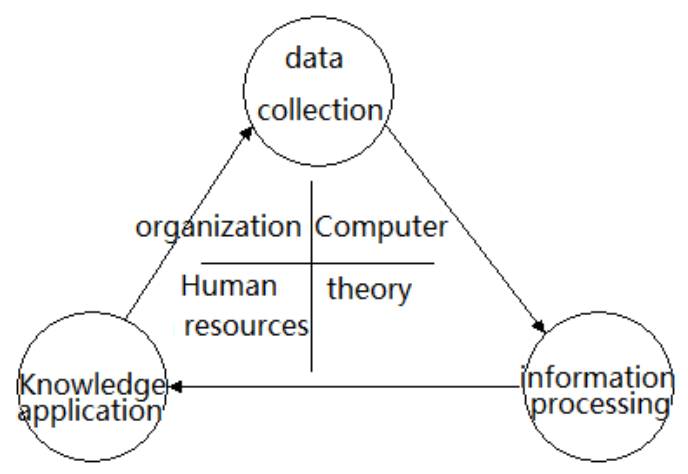

Fig.2 the continuous lifting cycle model of learning behavior analysis

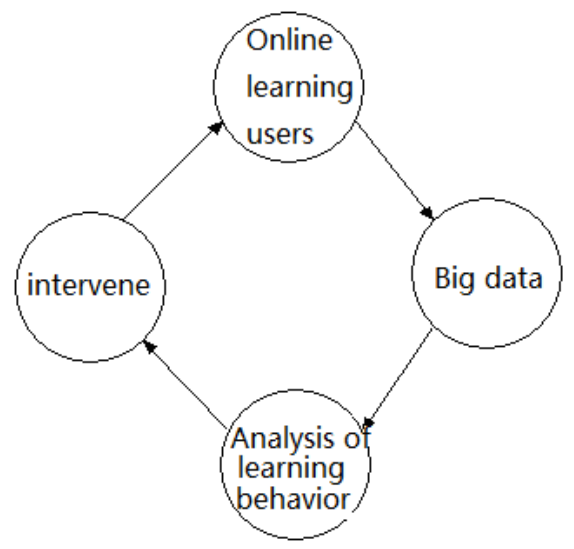

Fig.3 The cycle model for learning behavior analysis

As shown in Figure 3, the cyclic model of learning behavior analysis shows that the feedback of intervention measures is very important to the realization of the analysis of learning behavior. Of course, the intervention measures are not necessarily for the current learners and the later learners. After intervention, the analysis of learning behavior is carried out after intervention, and the learning knot before and after intervention is carried out. The comparison of the results, the formulation of a reasonable learning program, for the user to provide better guidance for online learning, but also for the network learning platform to provide better resources to lay a theoretical foundation.

\section{The online learning behavior analysis strategy based on big data}

The analysis of online learning behavior in large data environment can provide people with rules and learning strategies based on online learning behavior data and educational resources. It has important meaning for online learning users to improve learning efficiency and optimize the allocation of learning data.

Learning analysis can excavate the information and rules hidden behind the learning process of online learning users, and provide technical reference for online user learning results prediction, teaching intervention and learning individualization and self-adaptive. At present, the study of learning analysis is mainly concentrated in the stage of theoretical analysis, and the popularization of the practice is not much, mainly because the network teaching platform, the client and the teaching system are not uniform. Therefore, the unified standard should be considered when the learning behavior analysis strategy is designated.

In addition to the interoperability of data, a unified data dictionary can also realize the consistency of the analysis data of learning behavior. The development of online learning system can be followed by law. Using semantic analysis technology, the computer can deal with the analysis data of learning behavior better. The unified digital dictionary can realize the cross platform operation of learning analysis, and also help to improve the efficiency of mining analysis 
data mining, and finally provide more intelligent and adaptive learning experience for online learning users.

\section{Conclusion}

In order to improve the efficiency of users' online learning behavior analysis, the technology and tools of current learning behavior analysis are studied in the background of large data, and several common analytical techniques and tools are summarized. This paper summarizes the common learning behavior analysis model, combined with three models, such as process analysis model, continuous lifting cycle model, behavior analysis cycle model and so on. It puts forward the strategy of data interoperability, unified digital dictionary and other online user learning behavior analysis, which is important for the analysis and research of Online user learning behavior. The value of reference.

\section{Acknowledgement}

The work was supported by two subtopics from the science and technology research project of Jiangxi Provincial Department of Education in 2017 with the project number GJJ171099 and the project name Modeling and research of user online learning behavior analysis model in big data environment. One of the subtopics is Research on user online learning behavior analysis model under big data environment, and the another is Analysis framework design of user's online learning behavior under big data environment.

\section{References}

[1] Hu Dewei.big data "revolution" education [N]. Guangming Daily, 2013-10-9 (5).

[2] Jiang Qiang, Zhao Yu, Wang Pengjiao. Empirical Study on two-way Adaptive Interaction Evaluation in adaptive learning system [J]. Modern distance education research, 2013, (5): 106-112.

[3] Li Changxian. Research on school users' network behavior classification system [J]. Statistics and management, 2013, 04 (061):144-145.

[4] Zhang Jinliang, Ho tall. Learning analysis: boosting the online professional development of university teachers in the era of big data [J]. Journal of distance education, 2014, (2): 56-62.

[5] Jiang Qiang, Zhao Yu, Wang Pengjiao. An adaptive learning system architecture based on GALSRM model [J]. Modern distance education, 2013, (1): 71-77.

[6] Jiang Qiang, Zhao Yu. The design and implementation of an adaptive learning system for the perspective of "service". [J]. audio-visual education in China, 2011, (2): 119-124.

[7] M.A.Chatti, A.L.Dyckhoff, U.Schroeder, H.Th U S. A Reference Model for Learning Analytics [J]. International. (1): 318-331

[8] Li Yanyan, Ma Shaoqian, Huang Ronghuai. Learning analysis technology: service learning process design and optimization of [J]. Open education research, 2012, (5): 18-24.

[9] Wang Shan, Wang Hui Ju, Qin Xiong, et al. Big data: challenges, status quo and Prospect [J]. Journal of computer science, 2011, 34 (10): 1741-1752.

[10] Luo Ping. Analysis and research of student behavior in campus network in the age of big data [J]. Fujian computer, 2014, 10:147-148.

[11] Qin Xiong pie, Wang Hui Ju, Du Xiaoyong, et al. Big data analysis of a RI) BMS and MapReduce competition and symbiosis [J].2012.23 (1): 32-45.

[12] Liu Kan, Zhou Xiaozheng. Data visualization research and development [J]. Computer Engineering, 2002, 28 (8): 1-2. 
[13] Liu Mengchao, Xiao Jirong, Chen Rong. Application of data mining in user behavior analysis. Computer knowledge and technology, 2012, 31 (04): 7409-7412.

[14] Pan Feng Nan. Analysis and Research on user behavior of campus network [J]. Journal of Yangtze University, 2013, 23 (02): 1403-1409. 